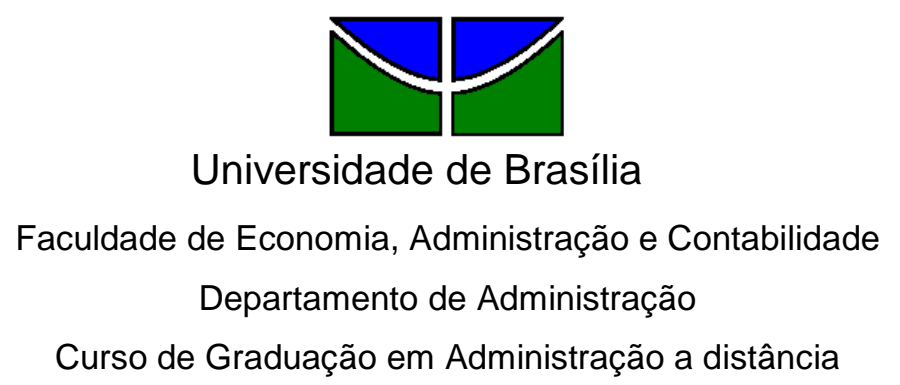

MICHEL FÉLIX DE MOURA

\title{
Análise da Eficiência do Fluxo de Dividendos Descontados Como Método de Avaliação de Ativos
}

Brasília - DF 


\section{MICHEL FÉLIX DE MOURA}

\section{Análise da Eficiência do Fluxo de Dividendos Descontados Como Método de Avaliação de Ativos}

Monografia apresentada a Universidade de Brasília (UnB) como requisito parcial para obtenção do grau de Bacharel em Administração.

Professor Orientador: Wolney Resende de Oliveira 
De Moura, Michel Félix.

Análise da Eficiência do Fluxo de Dividendos Descontados Como Método de Avaliação de Ativos / Michel Félix de Moura.-Brasília, 2010.

$30 \mathrm{f}$.

Monografia (bacharelado) - Universidade de Brasília, Departamento de Administração - EaD, 2010.

Orientador: Prof. Wolney Resende de Oliveira, Departamento de Administração.

1. Valuation. 2. Precificação de ativos. 3. Desconto de dividendos . I. Título. 


\section{MICHEL FÉLIX DE MOURA}

\section{Análise da Eficiência do Fluxo de Dividendos Descontados Como Método de Avaliação de Ativos}

A Comissão Examinadora, abaixo identificada, aprova o Trabalho de Conclusão do Curso de Administração da Universidade de Brasília do aluno

\section{MICHEL FÉLIX DE MOURA}

Prof. Wolney Resende de Oliveira

Professor-Orientador

Dr. José Carneiro da Cunha O. Neto

Professor-Examinador

\section{Prof. Wilian Gomes Xavier} Professor-Examinador 


\section{RESUMO}

É difícil mensurar o valor real de um ativo negociado em bolsa, determinando se sua cotação está acima ou abaixo do que seria o preço "justo" para aquisição/venda do papel. Existem alguns modelos para se determinar este preço justo, sendo o mais utilizado atualmente o método de fluxo de caixa descontados. Todavia, este método é de difícil aplicação para o investidor iniciante em bolsa. Um método de aplicação mais imediata seria de maior utilidade para o investidor pessoa física, facilitando os cálculos e dando uma alternativa de avaliação de empresas segundo os critérios fundamentalistas. Com isso, vamos testar neste trabalho a eficiência do método de fluxo de dividendos descontados ao valor presente, pois é de mais fácil aplicação. Assim, pretendemos determinar se este método é eficiente para uso e aplicação em ações da BOVESPA.

Palavras-chave: 1.Valuation. 2.Desconto de dividendos. carteiras. 


\section{LISTA DE ILUSTRAÇÕES}

Figura 1 - Comparativo carteiras x IBOV .29 


\section{LISTA DE TABELAS}

Tabela 1 - Exemplo arquivo dividendos Bovespa.............................................18

Tabela 2 - Dividendos agrupados por ano............................................... 18

Tabela 3 - Papéis da amostra ...........................................................20

Tabela 4 - Variação pagamento de dividendos ............................................... 21

Tabela 5 - SELIC anual ............................................................................ 22

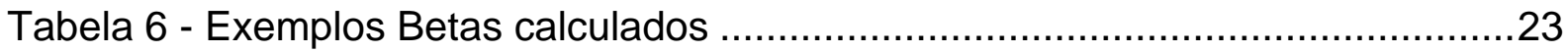

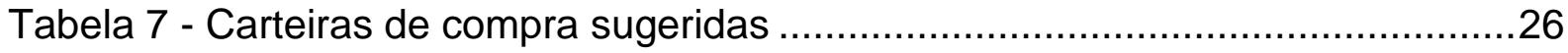

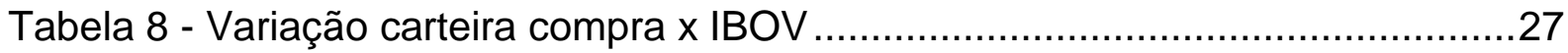

Tabela 9 - Variação carteira sobrevalorizada ................................................28 


\section{SUMÁRIO}

1 INTRODUÇÃO

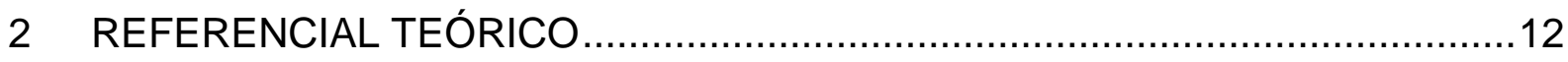

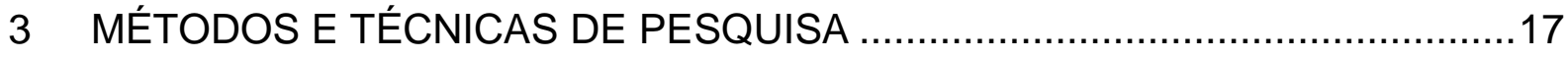

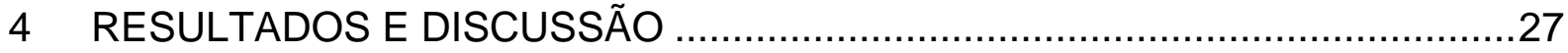

5 CONCLUSÕES E RECOMENDAÇÕES ………......................................

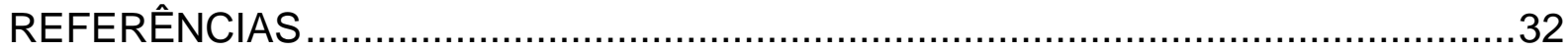




\section{INTRODUÇÃO}

Ao longo dos anos, vários métodos de avaliação de ativos têm sido usados para determinar o valor justo de uma empresa, ou seja, o quanto ela vale no mercado. $A$ maior parte destes métodos leva em conta o fluxo descontado, o seja, faz uma previsão futura de quanto de caixa que a empresa geraria para depois trazer o valor para o presente, determinando o real valor da empresa. Dentre os modelos mais usados no mercado, temos o modelo de fluxo de caixa descontados e o modelo de fluxo de dividendos.

Neste trabalho, pretendemos avaliar o método de fluxo de dividendos descontados nas ações da bolsa de valores de São Paulo ( BOVESPA), determinando assim se um investidor que tivesse utilizado este método no passado, obteria sucesso em seus investimentos, e com isso, demonstrar a sua eficácia e aplicabilidade. Para isso, utilizaremos dados das empresas listadas na Bovespa como os relatórios, gráficos e balanços patrimoniais.

O modelo de crescimento de Gordon pode ser usado para avaliar a empresa que está em "estado estável", com os dividendos crescendo a uma taxa que se espera permaneça estável a longo prazo (DAMODARAN, 1997). Este modelo baseia-se no cálculo de fluxo de dividendos e faz algumas suposições, como uma evolução constante do lucro da empresa, de acordo com a fórmula abaixo.

$$
P o=\frac{D t}{K-G}
$$

Onde:

Po = Preço atual da ação

Dt = Dividendos para o próximo período

$\mathrm{K}=$ Taxa de retorno exigida para o investimento

$\mathrm{G}=$ Taxa de crescimento para os dividendos

Com o uso da metodologia de Gordon, pretendemos avaliar se um investidor que tivesse utilizado esta metodologia conseguiria êxito em seus investimentos. 
Segundo a atual legislação brasileira, as empresas devem fazer um pagamento mínimo de dividendos a ser definido em seu estatuto. Por exemplo, o Banco do Brasil em seu estatuto, artigo 44, apresenta a seguinte informação:

Art. 44. Aos acionistas é assegurado o recebimento semestral de dividendo mínimo e obrigatório equivalente a $25 \%$ (vinte e cinco por cento) do lucro líquido ajustado, como definido em lei e neste Estatuto.

Ou seja, de acordo com o estatuto, os acionistas terão direito a um pagamento mínimo de $25 \%$ do lucro. Em geral as empresas pagam pelo menos $25 \%$ dos lucros como índice de payout. Baseado nisso, podemos calcular como foi a evolução do lucro nos anos anteriores e assim fazer uma estimativa de crescimento do lucro, permitindo avaliar a performance dos ativos baseado no modelo de Gordon.

\subsection{Formulação do problema}

Os preços das ações em bolsa se comportam de maneira aparentemente aleatória. Sabemos que vários fatores afetam o rumo das cotações em bolsa, como o ânimo dos investidores, o aquecimento da economia, a demanda interna e externa. Dentro de segmentos específicos podemos destacar a variação do preço de comodities, inflação, taxas de câmbio, etc. O que o investidor tem de concreto no final das contas é o balanço da empresa, que mostra a foto de tudo que ela produziu/gerou e o quando lucrou no período, e o quanto vai pagar de dividendos.

Muitos métodos são utilizados por investidores em todo o mundo para diminuir um pouco esta "aleatoriedade" e incerteza de preços. Dentre os investidores de bolsa, podemos destacar os que utilizam a análise técnica, ou análise gráfica, e os que utilizam métodos fundamentalistas. Cada uma destas áreas por si só é bastante ampla, mas, dentro da análise fundamentalista, vemos que o modelo de fluxo de caixa descontado é o mais utilizado para se avaliar uma empresa. Um modelo menos utilizado é o de fluxo de dividendos descontados, talvez por ser considerado de menor eficiência. Mas, seria mesmo este método ruim? Deveria ser relegado a 
um segundo plano? Respondendo a estas perguntas, podemos adicionar em nosso portfólio um indicador que pode melhorar o processo decisório do administrador financeiro.

\subsection{Objetivo Geral}

Como objetivos gerais do trabalho, iremos avaliar o modelo de fluxo de dividendos descontados e sua eficiência de utilização como método de avaliação de ativos e importância para formação de carteiras de dividendos. Para isso vamos selecionar todas as ações negociadas na Bovespa que fazem pagamentos de dividendos regularmente e fazer um comparativo de seus retornos usando o método de Gordon para cálculo do fluxo de dividendos descontados. Com isso, esperamos verificar se o preço do ativo no mercado está coerente com o valor em bolsa, gerando pontos de compra ou venda, e analisar se esta decisão foi coerente com a evolução do preço do papel no mercado.

Iremos simular um investidor que utilizou tal metodologia ao longo dos últimos 11 anos e verificar os retornos obtidos, sabendo assim se a metodologia foi útil na obtenção de bons resultados e ganhos financeiros.

\subsection{Objetivos Específicos}

Para conseguirmos avaliar os papéis, precisamos conseguir um histórico de período razoável em bolsa, de forma a diminuir a discrepância dos cálculos.

Selecionaremos ações negociadas na Bovespa com histórico de pagamentos de dividendos de pelos menos os 5 anos anteriores, em seguida, verificaremos 0 montante pago em título de dividendos ano a ano, calculando em seguida o dividend Yield. Com o dividend Yield em mãos, vamos avaliar a evolução do pagamento de dividendos ano a ano para saber qual é a taxa média de crescimento de dividendos, um dos componentes da fórmula do modelo de Gordon para estimar o cálculo no modelo utilizando dividendos crescentes. Caso o dividendo se mantenha estável, utilizaremos a fórmula apropriada sem a evolução dos dividendos. 
Para concluir, iremos montar as carteiras com preços subvalorizados comparando-a com o IBOV, e verificar a eficácia deste método.

\subsection{Justificativa}

Conforme já mencionamos, o modelo de fluxo de caixa descontado é hoje considerado o mais importante método de avaliação de ativos, entretanto alguns autores discordam da aplicação deste método na análise de valuation de uma empresa. SANTOS e PAMPLONA(2005) em seu artigo intitulado "Teoria das Opções Reais: uma atraente opção no processo de análise de investimentos” citando DIXIT e PINDYCK frisam que "... o fluxo de caixa descontado (FCD) não está somente errado, ele está muito errado". Neste caso os autores afirmam que o fluxo de caixa descontado não é capaz de assimilar a flexibilidade administrativa que permite ao gestor tomar uma decisão agora ou postergar para um momento mais oportuno, diminuindo o risco. Neste trabalho, não pretendemos desmerecer o fluxo de caixa descontado, mas apenas mostrar que existem outras opções de avaliação que também podem ser úteis, mais precisamente o fluxo de dividendos descontado, que permite avaliar uma empresa de acordo com o seu histórico de pagamento de dividendos de uma maneira mais prática que a empregada no fluxo de caixa.

O modelo de dividendos descontados é de mais fácil aplicação para o investidor comum e amplamente utilizado por investidores considerados "value investors", que fazem aplicações de longo prazo, no entanto, este método tem sido pouco utilizado e relegado a menor importância. 


\section{REFERENCIAL TEÓRICO}

Nenhum modelo fornece um valor preciso e único para avaliação de uma empresa, mas sim, uma estimativa de valor (MARTELANC, 2005). O que os modelos fazem é uma tentativa de estimar o valor mais aproximado possível da empresa de forma a diminuir os riscos do investimento. Dependendo do modelo adotado, das premissas e dos cenários criados, dois avaliadores podem obter valores diferentes para a mesma empresa (IBIDEM), isso porque é impossível não levar em conta valores pessoais que são atribuídos as suas variáveis de análise, que levam em conta várias suposições, como por exemplo, o retorno exigido ao nível de risco daquele ativo.

Segundo o mesmo autor (2005):

... em uma pesquisa feita pela associação dos analistas profissionais de investimento do mercado de Capitais ( APIMEC) em 2001, 88\% dos avaliadores utilizam o método do fluxo de caixa descontado em suas avaliações e $82 \%$ usam o método dos múltiplos. De acordo com essa mesma pesquisa, a utilização de uma única abordagem é preferida por apenas $23 \%$ dos avaliadores, ao passo que $60 \%$ preferem usar duas abordagens, e $18 \%$, três

Com isso, podemos ver a preferência dos analistas, mas vemos também que a maioria prefere utilizar mais de um indicador de referência. Ativos financeiros são adquiridos pelos fluxos de caixa esperados em decorrência de sua posse. Consequentemente, percepções de valor têm que ter o suporte da realidade, o que implica que o preço pago por qualquer ativo deve refletir os fluxos de caixa que se espera sejam por ele gerados.(DAMODARAN, 1997)

Uma das abordagens para cálculo de valor de mercado de uma empresa é pelo valor pago em dividendos aos seus acionistas.

Segundo o autor brasileiro, Décio Bazin (1992), a única razão para se comprar uma ação (ou uma empresa) é o retorno esperado pelos dividendos que a empresa pode gerar, ou seja, os dividendos são o motivo para se adquirir uma empresa, esperando-se obter um retorno sobre o investimento, e este deve ser acima dos valores pagos em títulos livres de risco. Segundo a autora Mary Buffet (2010), que 
conviveu durante um longo tempo com o "oráculo de Omaha" Warren Buffet, o valuation é também o método preferido e o unicamente utilizado por ele.

O recebimento de dividendos é um dos critérios utilizados por investidores em todo 0 mundo, por uma variedade de motivos diferentes, mas principalmente, como uma fonte de renda.

Há algumas evidências de que políticas de dividendos diferentes atraem tipos distintos de investidores, ou seja, clientelas diferentes. Este argumento sugere que as empresas com índices de distribuição elevados e estáveis atraem os investidores que preferem receber uma proporção significativa de seu retorno total sob a forma de rendimentos correntes. Tais investidores podem muito bem depender de dividendos para seu consumo corrente e, portanto, podem preferir um dividendo estável e previsível. (SOLOMON,1981)

O valor que a empresa paga em dividendos influencia diretamente o valor de seus papéis. Evidências empíricas (FERNÁNDEZ, 2002) mostram que empresas que pagam mais dividendos ( Maior porcentagem dos lucros ) não obtém um crescimento no preço das ações como resultado. Isso porque quando uma empresa distribui mais dividendos, normalmente reduz seu crescimento porque distribui o dinheiro aos acionistas em vez de colocá-lo em novos investimentos (IBIDEM)

O valor da ação ordinária de uma empresa uma empresa para o investidor é igual ao valor presente de todos os dividendos futuros esperados ( Ross, 1995 ). Vemos isso conforme a fórmula abaixo:

$$
P=\sum_{t=1}^{\infty} \frac{D t}{\left(1+k_{i}\right)^{t}}
$$

Ou seja, devemos trazer para valor presente, os valores previstos de pagamento de dividendos futuros. O modelo acima pode ser simplificado conforme demonstrado por Fernández (2002) na seguinte fórmula para o caso de não crescimento dos lucros:

$$
P o=\frac{D t}{K}
$$

Onde:

Po = Preço atual da ação 
Dt = Dividendos para o próximo período

$\mathrm{K}=$ Taxa de retorno exigida para o investimento

Neste, que é o modelo de Gordon, não é considerado um crescimento perpétuo para o pagamento dos dividendos.

O modelo de Gordon baseia-se no cálculo de fluxo de dividendos e faz algumas suposições, como uma evolução constante do lucro da empresa (podendo ser nulo), de acordo com a fórmula abaixo.

$$
P o=\frac{D t}{K-G}
$$

Onde:

$G=$ Taxa de crescimento para os dividendos

O Elemento K ( taxa de retorno ou custo de capital próprio) é um dos importantes elementos no cálculo do preço justo do ativo. Segundo Martelanc ( 2002 ) "O custo de capital é a taxa de retorno mínima necessária para atrair capital para um investimento... Também pode ser entendido como a taxa que o investidor pode obter em outro investimento de risco semelhante" e ainda "... o custo de capital é o retorno mínimo que o projeto deverá fornecer para conseguir captar o capital necessário para se viabilizar".

Vemos que o custo de capital é uma taxa de retorno exigida para os investidores aplicarem naquele investimento, sendo um importante fator a ser determinado, todavia, não está livre de incertezas e influências de cada analista que atribui riscos de acordo com seus critérios. Solomon (1981) faz algumas considerações interessantes:

Num mundo de incerteza, a relação apropriada de troca entre uma quantia no presente e uma expectativa de receber essa quantia no futuro não incluirá apenas o valor do dinheiro no tempo, mas uma valor adicional para cobrir a incerteza existente(...) O custo de capital é a taxa de retorno exigida por aqueles que fornecem o capital.

Ou seja, qual seria esta taxa de retorno adequada?

Utilizando o critério sugerido pelo autor, deveríamos então somar um valor do risco de investir naquele papel (incertezas ) ao valor que obteríamos digamos, investindo em títulos livres de risco (valor do dinheiro no tempo). 
Uma das dificuldades em se medir o custo de capital em países emergentes é a alta oscilação da bolsa nesses países, associado a incertezas políticas e de interferência do governo nesses mercados, que faz com que a taxa requerida se torne mais alta que em países mais desenvolvidos e mercados mais estáveis. Copeland (2000) sugere que com o tempo, os países se tornarão mais homogêneos, eliminando essas barreiras devido ao mercado globalizado:

A premissa básica de toda esta seção é que, com o tempo, os países cada vez mais farão parte de um mercado global e que esses mercados se tornarão mais abertos e eficientes. Embora o CAPM não funcione particularmente bem em muitos mercados emergentes, acreditamos que, ainda assim, oferece a melhor estimativa do retorno exigido pelo capital acionário em todo o mundo. Esperamos que as diferenças nos betas das indústrias locais e globais diminuam. Finalmente, esperamos que controles sobre os fluxos de capitais que restringem o acesso ao capital a custos internacionais sejam gradualmente reduzidos. O resultado é que o custo de capital nos mercados emergentes deverá se aproximar do custo de capital global após ajustes pela inflação local e pela estrutura de capital da empresa.

De acordo com o modelo de crescimento de Gordon, vemos que existem situações adequadas para a utilização da metodologia. A fórmula deve ser aplicada em empresas que têm um histórico regular de pagamento de dividendos. Devemos atentar particularmente para dois casos extremos quando se estima $\mathrm{K}$ para títulos individuais (ROSS,1995).

Primeiro, considere-se o caso de uma empresa que não está distribuindo lucros. O preço da ação será superior a zero, porque os investidores acreditam que a empresa começará a pagar um dividendo mais cedo ou mais tarde, ou porque a empresa será adquirida no futuro. Entretanto, quando uma empresa passa do não pagamento de dividendos para um dividendo positivo, a taxa de crescimento implícita é infinita. Por conseguinte, a equação deve ser utilizada com extremo cuidado neste caso, ou deve ser deixada de lado...

Em segundo lugar, mencionamos anteriormente que o valor da empresa tende a infinito quanto $\mathrm{g}$ tende a $\mathrm{k}$. Como os preços de ações não se comportam desse modo, um analista cuja estimativa de g pra uma dada 
empresa é igual ou superior a $\mathrm{k}$ deve ter cometido algum erro. $\mathrm{O}$ mais provável é que a elevada estimativa de g desse analista esteja correta para os anos mais próximos. Entretanto, as empresas simplesmente não podem manter uma taxa extraordinariamente alta de crescimento para sempre. 0 erro do analista foi utilizar uma estimativa de $\mathrm{g}$ para o curto prazo num modelo que requer uma taxa de crescimento perpétuo.

Ou seja, de acordo com Ross, devemos atentar para empresas que não estão pagando dividendos, ou estão tendo prejuízos, isso não significa que a ação não tenha valor, mas apenas que algum outro método de avaliação deve ser utilizado, e em segundo lugar, deve-se ter cuidado com taxas de crescimento muito grande que podem fazer com que o preço da ação tenda para valores muito altos. Damodaran (1997) observa ainda que:

Taxas passadas de crescimento são úteis na previsão de crescimento futuro, mas raramente podem ser consideradas como informação suficiente. Num estudo do relacionamento entre taxas de crescimento passadas e taxas de crescimento futuras, Little (1960) cunhou o termo "crescimento desordenado", porque descobriu poucas evidências de que empresas que cresceram rapidamente num período continuaram a crescer rapidamente no período seguinte. No processo de testar uma série de correlações entre taxas de crescimento em períodos consecutivos de diferentes extensões, ele freqüentemente encontrou correlações negativas entre taxas de crescimento em dois períodos, e a correlação média nos dois períodos era próxima de zero.

Em resumo, o modelo de crescimento de Gordon se ajusta melhor a empresas que crescem a uma taxa comparável ou inferior à taxa nominal de crescimento da economia, e que tenham políticas de pagamento de dividendos em relação aos lucros bem estabelecidas e que pretendam continuá-las a executar no futuro (DAMODARAN, 1997). O payout de dividendos em relação aos lucros da empresa tem de ser consistente com a hipótese de estabilidade, pois empresas estáveis geralmente pagam dividendos substanciais. (IBIDEM) 


\section{MÉTODOS E TÉCNICAS DE PESQUISA}

Para definirmos o método de pesquisa utilizado neste trabalho, devemos voltar a uma análise do problema proposto, como afirma Cervo - Metodologia Científica, $6^{\underline{a}}$ edição - o método de pesquisa depende fundamentalmente do objeto da pesquisa.

Vamos então colocar novamente o problema da pesquisa com algumas perguntas: Seria o valor do fluxo de dividendos importante para a avaliação de uma empresa? Esse cálculo é realmente útil na determinação do preço justo de um papel? Podemos empregar com uma certa margem de garantia este método, tornando assim a operação menos arriscada? Será este método melhor que o mais usual "fluxo de caixa descontado"?

Como iremos testar a eficiência do fluxo de dividendos descontados como método de avaliação de ativos para motivar a compra ou venda de determinado papel precisamos saber quais foram os dividendos pagos pelas empresas listadas na bolsa. Vamos fazer uma análise geral de todas as empresas sem tender para nenhuma ou outra com maior ou menor preferência pelos analistas de mercado, vamos olhar unicamente os dividendos pagos que é o critério de compra/venda para este trabalho.

No site da Bovespa(2010), conseguimos o arquivo com todos os pagamentos de dividendos feitos pelas companhias listadas na bolsa desde 1996. Este arquivo é gigantesco e possui mais de 11.500 lançamentos de proventos, e é a fonte principal de dados para essa pesquisa. Segue abaixo pequeno exemplo dos dados apresentados no arquivo da Bovespa para as ações do Banco do Brasil.

$\begin{array}{lccllrr}\begin{array}{c}\text { Nome } \\ \text { de } \\ \text { Pregão }\end{array} & \begin{array}{c}\text { Tipo } \\ \text { da } \\ \text { Ação }\end{array} & \begin{array}{c}\text { Data da } \\ \text { Aprovação }\end{array} & \begin{array}{l}\text { Valor do } \\ \text { Provento R\$ }\end{array} & \text { Tipo do Provento } & \begin{array}{c}\text { Último } \\ \text { Preço } \\ \text { "Com" }\end{array} & \begin{array}{c}\text { Provento/ } \\ \text { Preço \% }\end{array} \\ \text { BRASIL } & \text { ON } & 29 / 9 / 1997 & 0,112500000 & \text { DIVIDENDO } & 10,24 & 1,098633 \\ \text { BRASIL } & \text { ON } & 6 / 4 / 1998 & 0,112980000 & \text { DIVIDENDO } & 11,00 & 1,027091 \\ \text { BRASIL } & \text { ON } & 3 / 8 / 1998 & 0,157710000 & \text { JRS CAP PRÓPRIO } & 9,50 & 1,660105 \\ \text { BRASIL } & \text { ON } & 8 / 2 / 1999 & 0,176270000 & \text { JRS CAP PRÓPRIO } & 7,01 & 2,514551 \\ \text { BRASIL } & \text { ON } & 26 / 7 / 1999 & 0,215870000 & \text { JRS CAP PRÓPRIO } & 6,25 & 3,453920 \\ \text { BRASIL } & \text { ON } & 26 / 7 / 1999 & 0,003109700 & \text { RENDIMENTO } & 6,25 & 0,049755 \\ \text { BRASIL } & \text { ON } & 17 / 3 / 2000 & 0,101060000 & \text { JRS CAP PRÓPRIO } & 6,80 & 1,486176 \\ \text { BRASIL } & \text { ON } & 17 / 3 / 2000 & 0,003750000 & \text { RENDIMENTO } & 6,80 & 0,055147 \\ \text { BRASIL } & \text { ON } & 7 / 8 / 2000 & 0,147880000 & \text { JRS CAP PRÓPRIO } & 6,41 & 2,307020 \\ \text { BRASIL } & \text { ON } & 7 / 8 / 2000 & 0,002420000 & \text { RENDIMENTO } & 6,41 & 0,037754\end{array}$




$\begin{array}{lllllll}\text { BRASIL } & \text { ON } & 12 / 2 / 2001 & 0,199160000 & \text { DIVIDENDO } & 7,75 & 2,569806 \\ \text { BRASIL } & \text { ON } & 12 / 2 / 2001 & 0,003420000 & \text { RENDIMENTO } & 7,75 & 0,044129 \\ \text { BRASIL } & \text { ON } & 24 / 8 / 2001 & 0,103646800 & \text { DIVIDENDO } & 9,44 & 1,097953\end{array}$

Tabela 1 - Exemplo arquivo dividendos Bovespa

Para calcularmos o retorno da ação durante o ano, devemos fazer a soma de todo o dividendo pago durante o ano, então, agrupamos todos os proventos feitos durante o ano em uma única linha por ano, desta forma, poderemos ver quais as ações que pagam dividendos regularmente, pois este é um dos princípios para se investir baseado em dividendos, que haja uma constância nos pagamentos. Desta forma, a tabela passou a ter 6.067 registros. Seguem abaixo os lançamentos do Banco do Brasil agrupados por ano como exemplo.

Tabela 2 - Dividendos agrupados por ano

\begin{tabular}{|l|l|l|r|r|r|}
\hline ATIVO & TP & \multicolumn{1}{|c|}{ TP_PVT } & ANO & DIV_PAGOS & \multicolumn{1}{c|}{ YIELD } \\
\hline BRASIL & ON & DIVIDENDO & 1997 & 0,1125 & 1,098633 \\
\hline BRASIL & PN & DIVIDENDO & 1997 & 0,1237 & 0,998386 \\
\hline BRASIL & ON & DIVIDENDO & 1998 & 0,11298 & 1,027091 \\
\hline BRASIL & ON & JRS CAP PRÓPRIO & 1998 & 0,15771 & 1,660105 \\
\hline BRASIL & PN & DIVIDENDO & 1998 & 0,12428 & 0,836902 \\
\hline BRASIL & PN & JRS CAP PRÓPRIO & 1998 & 0,17348 & 1,344806 \\
\hline BRASIL & ON & JRS CAP PRÓPRIO & 1999 & 0,39214 & 5,968471 \\
\hline BRASIL & PN & JRS CAP PRÓPRIO & 1999 & 0,43136 & 4,975589 \\
\hline BRASIL & ON & JRS CAP PRÓPRIO & 2000 & 0,24894 & 3,793197 \\
\hline BRASIL & PN & JRS CAP PRÓPRIO & 2000 & 0,27382 & 2,950597 \\
\hline BRASIL & ON & DIVIDENDO & 2001 & 0,3028068 & 3,66776 \\
\hline BRASIL & PN & DIVIDENDO & 2001 & 0,3330814 & 3,324659 \\
\hline BRASIL & ON & DIVIDENDO & 2002 & 0,6670194 & 6,061255 \\
\hline BRASIL & PN & DIVIDENDO & 2002 & 0,73373134 & 6,141848 \\
\hline BRASIL & ON & DIVIDENDO & 2003 & 0,37167 & 3,784827 \\
\hline BRASIL & ON & JRS CAP PRÓPRIO & 2003 & 0,43977 & 2,808238 \\
\hline BRASIL & ON & JRS CAP PRÓPRIO & 2004 & 1,54258 & 6,09221 \\
\hline BRASIL & ON & JRS CAP PRÓPRIO & 2005 & 1,06776 & 2,975183 \\
\hline BRASIL & ON & DIVIDENDO & 2006 & 2,160536 & 4,077192 \\
\hline BRASIL & ON & JRS CAP PRÓPRIO & 2006 & 2,600345 & 4,603199 \\
\hline BRASIL & ON & DIVIDENDO & 2007 & 0,637604556 & 1,130439 \\
\hline BRASIL & ON & JRS CAP PRÓPRIO & 2007 & 0,797274026 & 1,96412 \\
\hline BRASIL & ON & DIVIDENDO & 2008 & 0,527373219 & 2,43241 \\
\hline BRASIL & ON & JRS CAP PRÓPRIO & 2008 & 0,607554457 & 2,890142 \\
\hline BRASIL & ON & DIVIDENDO & 2009 & 0,695331491 & 3,724554 \\
\hline BRASIL & ON & JRS CAP PRÓPRIO & 2009 & 0,723699807 & 3,186985 \\
\hline BRASIL & ON & DIVIDENDO & 2010 & 0,831500187 & 2,857527 \\
\hline
\end{tabular}


Neste trabalho, iremos delimitar o período de análise dos retornos sobre os dividendos para o período de 2004 à 2009, ou seja, dos últimos 5 anos excluindo o ano de 2010 que ainda não está com o calendário de dividendos fechado. Mas para tratar um período de 5 anos, vamos ter que retroagir nos eventos de 10 anos atrás pois é necessário fazermos uma média histórica de pelo menos 5 anos antes para o investidor que começou a aplicar segundo este critério em 2004, então vamos restringir na nossa tabela os registros de dividendos entre 1998 e 2009, e destarte, nos restaram 3341 linhas para serem analisadas.

Para efeito de cálculo de dividendos, os lançamentos de DIVIDENDO e JUROS SOBRE O CAPITAL PRÓPRIO devem se somar. Fizemos este novo agrupamento e nos sobraram 2458 linhas na tabela.

Agora temos uma tabela com todas as empresas que pagaram dividendos entre 1998 e 2009, só que a análise de dividendos descontados prevê que a empresa tenha uma constância neste pagamento pois é esperado que o investidor tenha 0 seu "rendimento" anualmente para a utilização do modelo de Gordon (Ross,1995), devendo fazer-lhe ano a ano, de forma que o investidor possa ter uma renda certa com um determinado dividend yield. Com este critério, selecionamos as ações que pagaram dividendos pelo menos nos 5 anos anteriores, para então estimar para 0 próximo período o valor a receber de dividendos e a taxa de crescimento do mesmo. Temos agora o total de 173 papéis de empresas elegíveis para compor as carteiras de ações subavaliadas. Utilizaremos ainda um segundo critério de liquidez, pois uma ação que não tem liquidez torna muito difícil a sua aquisição, venda e precificação, tornando muito difícil para o investidor entrar no negócio com a cotação de "preço justo" calculada pelo sistema. 


\subsection{População e amostra do estudo}

Utilizando os critérios de pagamentos de dividendos constantes (pelo menos os últimos 5 anos) e liquidez (pelo menos 20 negócios no ano) chegamos as ações elegíveis para compor as carteiras com preços subavaliados para serem montadas no período de 2004 à 2009.

Abaixo temos os papéis que foram selecionados:

Tabela 3 - Papéis da amostra

\begin{tabular}{|c|c|c|c|c|c|}
\hline ALPA3 & BSLI3 & CSAB3 & FRAS3 & LUXM4 & RPMG3 \\
\hline ALPA4 & BSLI4 & CSAB4 & FRAS4 & MERC3 & RPMG4 \\
\hline APTI4 & CEDO3 & CSNA3 & GGBR3 & MERC4 & RSID3 \\
\hline BAHI3 & CEEB3 & CTKA3 & GGBR4 & MGEL4 & SBSP3 \\
\hline BAZA3 & CEEB5 & CTKA4 & GLOB11 & MOAR3 & SLED3 \\
\hline BBAS3 & CEPE3 & CTNM3 & GLOB3 & MRSL4 & SLED4 \\
\hline BBDC10 & CEPE5 & CTNM4 & GLOB4 & MTIG3 & SOND3 \\
\hline BBDC3 & CEPE6 & CYRE3 & GOAU3 & MTIG4 & SOND5 \\
\hline BBDC4 & CGAS3 & DOHL4 & GOAU4 & MTSA4 & SOND6 \\
\hline BMEB3 & CGAS5 & DROG3 & GUAR3 & NAFG4 & TCNO3 \\
\hline BMEB4 & CGRA3 & DUQE3 & GUAR4 & PATI3 & TCNO4 \\
\hline BMIN3 & CGRA4 & DUQE4 & IGUA3 & PATI4 & TCSL3 \\
\hline BMIN4 & CIQU3 & DXTG4 & IGUA5 & PCAR10 & TCSL4 \\
\hline BMKS3 & CIQU4 & ELET3 & IGUA6 & PCAR11 & TKNO4 \\
\hline BMTO3 & CLSC3 & ELET5 & ITAU3 & PCAR3 & TLPP3 \\
\hline BMTO4 & CLSC6 & ELET6 & ITAU4 & PCAR4 & TLPP4 \\
\hline BNBR3 & CMIG3 & ELMJ3 & ITSA10 & PETR3 & UNIP3 \\
\hline BNBR4 & CMIG4 & ELMJ4 & ITSA3 & PETR4 & UNIP5 \\
\hline BRFS3 & CNFB3 & EMBR3 & ITSA4 & PNVL3 & UNIP6 \\
\hline BRGE11 & CNFB4 & EMBR4 & ITUB3 & PNVL4 & USIM3 \\
\hline BRGE12 & COCE3 & ETER3 & ITUB4 & POMO3 & USIM5 \\
\hline BRGE3 & COCE5 & ETER4 & JFEN3 & POMO4 & USIM6 \\
\hline BRGE5 & COCE6 & FBMC4 & JOPA3 & PRGA3 & VALE3 \\
\hline BRGE6 & CPLE3 & FESA3 & JOPA4 & PTNT4 & VALE5 \\
\hline BRIV3 & CPLE5 & FESA4 & KEPL3 & RAPT3 & WEGE3 \\
\hline BRIV4 & CPLE6 & FFTL3 & LAME3 & RAPT4 & WEGE4 \\
\hline BRTO3 & CRIV3 & FFTL4 & LAME4 & RPAD3 & WHRL3 \\
\hline BRTO4 & CRUZ3 & FJTA3 & LEVE4 & RPAD5 & WHRL4 \\
\hline
\end{tabular}




\subsection{Caracterização dos instrumentos de pesquisa}

Agora que já sabemos quais os papéis serão analisados, iremos fazer a estimativa de pagamento de dividendos para o próximo período, pois este dado é necessário para o uso na fórmula do modelo de Gordon, conforme mostrado por Damodaran(1997):

$$
P o=\frac{D t}{K-G}
$$

Onde:

Po = Preço atual da ação

Dt = Dividendos para o próximo período

$\mathrm{K}=$ Taxa de retorno exigida para o investimento

$\mathrm{G}=$ Taxa de crescimento para os dividendos

Segundo Gitman(2004) “... devemos verificar a evolução do crescimento dos dividendos" para a avaliação correta do ativo. Com isso, o parâmetro Dt, pagamento de dividendos para o próximo período será calculado com base na evolução do pagamento de dividendos daquele ativo nos 5 anos anteriores. Então fizemos o cálculo da evolução dos dividendos ano a ano para todas os ativos selecionados. Segue abaixo o exemplo da variação no pagamento de dividendos para as ações do Banco do Brasil em 1 e 5 anos.

Tabela 4 - Variação pagamento de dividendos

\begin{tabular}{|l|l|r|r|r|r|}
\hline \multicolumn{1}{|c|}{ ATIVO } & TP & ANO & \multicolumn{1}{c|}{ Div Pagos } & \multicolumn{1}{c|}{1 Ano } & 5 Anos \\
\hline BRASIL & ON & 2000 & 0,24894 & $-36,52 \%$ & \\
\hline BRASIL & ON & 2001 & 0,3028068 & $21,64 \%$ & \\
\hline BRASIL & ON & 2002 & 0,6670194 & $120,28 \%$ & \\
\hline BRASIL & ON & 2003 & 0,81144 & $21,65 \%$ & $15,65 \%$ \\
\hline BRASIL & ON & 2004 & 1,54258 & $90,10 \%$ & $44,02 \%$ \\
\hline BRASIL & ON & 2005 & 1,06776 & $-30,78 \%$ & $28,67 \%$ \\
\hline BRASIL & ON & 2006 & 4,760881 & $345,88 \%$ & $48,15 \%$ \\
\hline BRASIL & ON & 2007 & 1,434878582 & $-69,86 \%$ & $12,08 \%$ \\
\hline BRASIL & ON & 2008 & 1,134927676 & $-20,90 \%$ & $-5,95 \%$ \\
\hline BRASIL & ON & 2009 & 1,419031298 & $25,03 \%$ & $5,85 \%$ \\
\hline
\end{tabular}


Então o parâmetro Dt para 2004, por exemplo, será a soma dos dividendos pagos em 2003 ( $R \$ 0,81144)$ acrescida da média de crescimento dos últimos 5 anos $(15,65 \%)$, que dá o total de $\mathrm{R} \$ 0,933156$.

O parâmetro $\mathrm{K}$, taxa de retorno exigida para o investimento é mais complicado de calcular, e é também chamado de custo do capital ou custo do patrimônio líquido CPL. Este parâmetro é formado da seguinte maneira:

$K=T L R+($ Beta $x$ prêmio de ações sobre TLR)

Onde TLR é a taxa livre de risco no mercado, sendo utilizado o bônus para pagamento de títulos pelo governo (Taxa SELIC). Buscando no site do Banco Central do Brasil (2010), obtivemos os seguintes valores para a taxa SELIC que serão utilizados no cálculo pelo sistema.

\begin{tabular}{|r|r|}
\hline ANO & SELIC\% \\
\hline 2000 & 15,75 \\
\hline 2001 & 19,00 \\
\hline 2002 & 25,00 \\
\hline 2003 & 16,50 \\
\hline 2004 & 17,75 \\
\hline 2005 & 18,00 \\
\hline 2006 & 13,25 \\
\hline 2007 & 11,25 \\
\hline 2008 & 13,75 \\
\hline 2009 & 8,75 \\
\hline Tabela 5 - SELIC anual
\end{tabular}

O Beta também é um cálculo complexo em que deve ser feito o cálculo da variância do IBOV no mesmo período em que serão analisadas a covariância do papel e do IBOV. O Beta é dado pela COVARIÂNCIA dividida pela VARIÂNCIA do IBOV. Seguem abaixo alguns betas calculadas para utilização no sistema das ações do BANCO DO BRASIL, VALE e PETROBRÁS. 


\begin{tabular}{|r|l|c|c|c|}
\hline ANO & PAPEL & VAR IBOV & COVAR & BETA \\
\hline 2007 & BBAS3 & 2,97942131 & 2,9632137 & 0,9945602 \\
\hline 2007 & PETR4 & 2,97942131 & 2,9511868 & 0,9905235 \\
\hline 2007 & VALE5 & 2,97942131 & 3,7020971 & 1,2425558 \\
\hline 2008 & BBAS3 & 10,9220993 & 12,132394 & 1,1108115 \\
\hline 2008 & PETR4 & 10,9220993 & 11,586766 & 1,0608552 \\
\hline 2008 & VALE5 & 10,9220993 & 12,101736 & 1,1080046 \\
\hline 2009 & BBAS3 & 3,93853347 & 3,5493497 & 0,9011856 \\
\hline 2009 & PETR4 & 3,93853347 & 3,8281543 & 0,9719745 \\
\hline 2009 & VALE5 & 3,93853347 & 4,7934779 & 1,2170718 \\
\hline \multicolumn{3}{|c}{ Tabela 6 - Exemplos Betas calculados } \\
\hline
\end{tabular}

O prêmio de ações sobre TLR pode ser calculado subtraindo-se a variação do IBOV pela taxa SELIC daquele ano (NUNES,M.I,2004), mas esta fórmula pode gerar valores absurdos no Brasil devido ao alto valor da taxa SELIC que pode gerar valores negativos neste contexto sendo inapropriado para este caso. Preferimos utilizar a mesma metodologia utilizada por Damodaran(1997) em seu livro Avaliação de Investimentos que utiliza uma taxa fixa para cálculo de dividendos descontados. No livro vemos que a taxa utilizada para prêmio de risco nos EUA é de 5,5\% então acrescentamos mais $2 \%$ no nosso cálculo, algo semelhante à taxa de risco Brasil e chegamos ao valor de $7,5 \%$ de prêmio de ações sobre títulos sem risco.

Um outro elemento da fórmula é a taxa de crescimento perpétuo dos dividendos que deve ser subtraída do custo do patrimônio líquido calculado conforme acima.

Difícil é estimar a taxa de crescimento perpétuo, pois, segundo Damodaran (1997), uma empresa que cresce acima da taxa de crescimento da economia do país indefinidamente tende a englobar todo o PIB daquele país, sendo portanto, impossível uma empresa crescer muito acima da economia. Todavia é possível que uma empresa cresça por um período a taxas extraordinárias. Já para o lado ruim, não existe um limite inferior para o decréscimo dos dividendos, sendo possível que eles caiam indefinidamente até a empresa começar a dar prejuízo e ir diminuindo de tamanho até que suma do mercado. Em um estudo feito por Sorensen e Williamson (1985, apud, Damodaran 1997) no mercado americano de 1980, foi utilizada a taxa de crescimento perpétuo de $8 \%$ para todas as empresas analisadas indistintamente do setor ou qualquer outro fator. Vemos que o Brasil possui características muito diversas do mercado norte-americano, como por exemplo uma maior taxa de crescimento da economia, devido a ser um pais emergente com grande foco 
internacional, maiores instabilidades também, tanto econômicas quanto políticas, uma taxa de juros que é uma das maiores do mundo, uma inflação também mais alta que na maior parte do mundo e que está em um processo de contenção nos últimos anos, e uma bolsa de valores ainda com volumes muito pequenos frente àquele mercado, e que ainda está se desenvolvendo, tendo portanto, grandes oscilações. Todos estes fatores dificultam a aplicação do modelo, e por isso, devemos fazer considerações no cálculo desta taxa de crescimento.

Para empresas que não estão tendo crescimento nos dividendos, crescimento menor que zero, vamos considerar uma taxa perpétua de $6 \%$, pois o pagamento de dividendos destas empresas é constante e assim, existe uma alta probabilidade do papel retornar aos níveis anteriores. Esta taxa está bem conservadora, pois este valor não cobre nem o crescimento do PIB + a inflação e é menor que a utilizada pelos autores mencionados acima. Já para as empresas com um crescimento de dividendos positivo entre 0 e $8 \%$, vamos utilizar o valor de $8 \%$, idem ao método usado nos EUA no referido estudo. Para as empresas com um bom crescimento no pagamento de dividendos, entre 8 e 15\%, iremos utilizar a média de crescimento da empresa dos últimos 5 anos, ou seja, se foi de 10\%, Então para o crescimento perpétuo também será de $10 \%$. Já para os casos de crescimento médio nos últimos 5 anos superior a $15 \%$, então utilizaremos a taxa de $15 \%$ fixa, pois como vimos acima, não é provável que este crescimento se mantenha por muito tempo, visto que a economia não cresce tão rapidamente.

Com isso, colocamos os sistema para gerar o cálculo do preço justo dos ativos e fazer uma comparação com o valor teórico do papel para saber quais as carteiras de ativos a serem compradas entre os anos de 2004 e 2009.

\subsection{Procedimentos de coleta e de análise de dados}

$\mathrm{Na}$ etapa anterior determinamos o preço justo do papel, agora temos que comparar com o papel negociado em bolsa para saber se a ação está subavaliada ou sobrecomprada. Não podemos fazer a comparação direta com o preço do papel negociado em bolsa pois os papéis negociados em bolsa sofrem a ação de vários 
eventos que alteram o preço dos mesmos tais como desdobramentos e agrupamentos, ex-dividendos e JCP, bonificações, etc, que afetam os preços dos papéis. Entretanto, o arquivo fornecido pela BOVESPA com os eventos de pagamentos de dividendos e juros sobre o capital próprio fornecem o valor do Yield daquele pagamento, assim, temos como determinar o preço "teórico" do papel, ou seja, se uma ação pagou $R \$ 1,00$ de dividendos e o Yield informado pela bolsa é de $10 \%$, então o preço teórico do papel é $\mathrm{R} \$ 10,00$.

Baseado nesta metodologia, comparamos os preços teóricos dos papéis com o preço que deveria ser, baseado na projeção segundo o modelo de Gordon de crescimento perpétuo de dividendos explicada no item anterior e o sistema montou as carteiras para os anos de 2004 a 2009 conforme a seguir.

\begin{tabular}{|l|l|l|l|l|l|}
\hline \multicolumn{1}{|c|}{$\mathbf{2 0 0 4}$} & \multicolumn{1}{|r|}{$\mathbf{2 0 0 5}$} & \multicolumn{1}{|r|}{$\mathbf{2 0 0 6}$} & \multicolumn{1}{|c|}{$\mathbf{2 0 0 7}$} & \multicolumn{1}{|c|}{$\mathbf{2 0 0 8}$} & \multicolumn{1}{|c|}{$\mathbf{2 0 0 9}$} \\
\hline ALPA3 & ALPA3 & APTI4 & BBAS3 & BAZA3 & BMKS3 \\
\hline ALPA4 & ALPA4 & BRGE12 & BBDC10 & BNBR3 & BNBR3 \\
\hline BBAS3 & BBDC3 & BRTO3 & BMEB3 & BNBR4 & BNBR4 \\
\hline BMKS3 & BBDC4 & BRTO4 & BMEB4 & BRGE11 & BRGE11 \\
\hline BRGE6 & CEEB3 & CEEB3 & BMKS3 & BRGE6 & BRGE6 \\
\hline CEDO3 & CEEB5 & CGAS3 & BRGE6 & CEEB3 & BRIV3 \\
\hline CEEB3 & CEPE5 & CRIV3 & BRIV4 & CEEB5 & BRIV4 \\
\hline CEPE5 & CEPE6 & CSAB3 & CEEB3 & CEPE3 & CEPE5 \\
\hline CEPE6 & CIQU3 & CSAB4 & CEEB5 & CEPE5 & CEPE6 \\
\hline CIQU3 & CRIV3 & ETER3 & CEPE5 & CEPE6 & CLSC6 \\
\hline CIQU4 & CSAB3 & ETER4 & CEPE6 & CGAS3 & CNFB4 \\
\hline COCE3 & CSAB4 & FBMC4 & CGAS3 & CGAS5 & COCE3 \\
\hline COCE5 & DROG3 & FESA4 & CGAS5 & CLSC3 & COCE5 \\
\hline COCE6 & DUQE4 & FFTL4 & CMIG3 & CLSC6 & COCE6 \\
\hline CRIV3 & FESA4 & IGUA3 & CMIG4 & CMIG3 & CPLE3 \\
\hline CRUZ3 & FRAS4 & IGUA5 & COCE3 & CMIG4 & CPLE5 \\
\hline CSAB3 & GGBR3 & IGUA6 & COCE5 & COCE3 & CPLE6 \\
\hline CSAB4 & IGUA3 & ITSA3 & COCE6 & COCE6 & FBMC4 \\
\hline CSNA3 & IGUA5 & LEVE4 & CRIV3 & CRIV3 & GLOB3 \\
\hline CTKA3 & IGUA6 & MOAR3 & CRIV4 & CRIV4 & GUAR3 \\
\hline CTKA4 & ITSA3 & MTSA4 & CSAB3 & CSAB3 & GUAR4 \\
\hline DROG3 & MRSL4 & PATI3 & CSAB4 & CSAB4 & ITSA10 \\
\hline ELMJ3 & MTSA4 & PATI4 & CSNA3 & DXTG4 & LUXM4 \\
\hline ELMJ4 & PATI3 & RAPT3 & DROG3 & ELET5 & MGEL4 \\
\hline ETER3 & PATI4 & RAPT4 & FBMC4 & EMBR3 & MOAR3 \\
\hline ETER4 & PNVL3 & RPAD5 & FRAS3 & FBMC4 & RPAD5 \\
\hline FESA4 & PNVL4 & SOND5 & IGUA3 & MOAR3 & SOND3 \\
\hline FFTL3 & PRGA3 & SOND6 & IGUA5 & RAPT3 & SOND5 \\
\hline
\end{tabular}




\begin{tabular}{|l|l|l|l|l|l|}
\multicolumn{1}{|c|}{$\mathbf{2 0 0 4}$} & $\mathbf{2 0 0 5}$ & $\mathbf{2 0 0 6}$ & $\mathbf{2 0 0 7}$ & $\mathbf{2 0 0 8}$ & $\mathbf{2 0 0 9}$ \\
\hline FFTL4 & SOND5 & TKNO4 & IGUA6 & RAPT4 & SOND6 \\
\hline GOAU3 & SOND6 & TLPP3 & LEVE4 & RPAD5 & TLPP3 \\
\hline GUAR3 & TKNO4 & TLPP4 & MERC4 & SOND5 & TLPP4 \\
\hline GUAR4 & TLPP3 & USIM3 & MOAR3 & SOND6 & \\
\hline IGUA3 & TLPP4 & USIM5 & MTSA4 & TCNO3 & \\
\hline IGUA5 & WEGE4 & USIM6 & RAPT3 & TCNO4 & \\
\hline IGUA6 & & & RAPT4 & TCSL4 & \\
\hline ITSA10 & & & RPAD5 & TLPP3 & \\
\hline ITSA3 & & & SOND5 & USIM3 & \\
\hline ITSA4 & & & SOND6 & USIM5 & \\
\hline JFEN3 & & & TKNO4 & USIM6 & \\
\hline MTSA4 & & & TLPP3 & WEGE3 & \\
\hline PATI3 & & & TLPP4 & & \\
\hline PATI4 & & & USIM3 & & \\
\hline PNVL4 & & & USIM5 & & \\
\hline PRGA3 & & & USIM6 & & \\
\hline SOND5 & & & WEGE3 & & \\
\hline TKNO4 & & & WEGE4 & & \\
\hline TLPP3 & & & & & \\
\hline TLPP4 & & & & & \\
\hline USIM3 & & & & & \\
\hline USIM5 & & & & & \\
\hline USIM6 & & & & & \\
\hline VALE3 & & & & & \\
\hline VALE5 & & & & & \\
\hline
\end{tabular}

Tabela 7 - Carteiras de compra sugeridas 


\section{RESULTADOS E DISCUSSÃO}

Baseado na metodologia exposta anteriormente, verificamos como se performaram as carteiras montadas pelo sistema.

Um investidor que tivesse aplicado $R \$ 1.000,00$ reais na carteira de 2004 e mantivesse a atualização da carteira anualmente conforme sugerido, obteria ao final de 2009 o valor de $R \$ 5.074,02$, um rendimento muito bom de $407,40 \%$. Neste mesmo período, se o investidor tivesse aplicado no índice BOVESPA teria o valor de $\mathrm{R} \$ 3.084,55$, um rendimento de $208,45 \%$.

Vemos com isso, que o investidor teve um ganho adicional de $64,5 \%$ aplicando segundo este critério de dividendos. Seguem abaixo as tabelas comparativas ano a ano.

\begin{tabular}{|r|r|r|r|}
\hline \multicolumn{5}{|c|}{ CARTEIRA } \\
\hline ANO & VARIAÇÃO & $1.000,00$ & ACUMULADO \\
\hline 2004 & $57,14 \%$ & $1.571,36$ & $57,14 \%$ \\
\hline 2005 & $32,59 \%$ & $2.083,44$ & $108,34 \%$ \\
\hline 2006 & $-1,87 \%$ & $2.044,50$ & $104,45 \%$ \\
\hline 2007 & $83,11 \%$ & $3.743,77$ & $274,38 \%$ \\
\hline 2008 & $57,10 \%$ & $5.881,28$ & $488,13 \%$ \\
\hline 2009 & $-13,73 \%$ & $5.074,02$ & $407,40 \%$ \\
\hline
\end{tabular}

\begin{tabular}{|r|r|r|r|}
\hline \multicolumn{5}{|c|}{ IBOV } \\
\hline ANO & VARIAÇÃO & $1.000,00$ & ACUMULADO \\
\hline 2004 & $17,81 \%$ & $1.178,09$ & $17,81 \%$ \\
\hline 2005 & $27,71 \%$ & $1.504,54$ & $50,45 \%$ \\
\hline 2006 & $32,93 \%$ & $2.000,04$ & $100,00 \%$ \\
\hline 2007 & $43,65 \%$ & $2.873,09$ & $187,31 \%$ \\
\hline 2008 & $-41,22 \%$ & $1.688,70$ & $68,87 \%$ \\
\hline 2009 & $82,66 \%$ & $3.084,55$ & $208,45 \%$ \\
\hline
\end{tabular}

Tabela 8 - Variação carteira compra x IBOV

Podemos observar também alguns fatos interessantes. A carteira sugerida pelo sistema atingiu seu topo no ano de 2008 , em plena crise internacional, onde apenas nesse ano, teve um rendimento de $57,10 \%$, frente a uma baixa de $41,22 \%$ do IBOV. A carteira marcou uma variação de $488,13 \%$ ao final deste ano, atingindo seu pico, enquanto ao final deste ano, o IBOV estava apenas com $68,87 \%$ de alta acumulada. Todavia, no ano seguinte, o IBOV subiu $82,66 \%$ enquanto a carteira de dividendos 
sugerida caiu 13,73\%. Percebemos então, que uma carteira de dividendos é atrativa em períodos de crise, pois os investidores preferem ativos que remuneram seu capital, diminuindo os riscos do investimento, e em períodos de recuperação, aparentemente os investidores retornam aos papéis mais arriscados, vendendo suas posições nos papéis mais seguros.

O sistema gerou ainda, uma carteira de papéis sobrecomprados ou sobrevalorizados para o mesmo período, a qual também vemos sua performance abaixo.

\begin{tabular}{|c|r|r|r|}
\hline \multicolumn{5}{|c|}{ CARTEIRA } \\
\hline ANO & VARIAÇÃO & $1.000,00$ & ACUMULADO \\
\hline 2004 & $73,84 \%$ & $1.738,40$ & $73,84 \%$ \\
\hline 2005 & $16,11 \%$ & $2.018,40$ & $101,84 \%$ \\
\hline 2006 & $38,56 \%$ & $2.796,66$ & $179,67 \%$ \\
\hline 2007 & $38,87 \%$ & $3.883,85$ & $288,39 \%$ \\
\hline 2008 & $-14,56 \%$ & $3.318,18$ & $231,82 \%$ \\
\hline 2009 & $0,80 \%$ & $3.344,56$ & $234,46 \%$ \\
\hline
\end{tabular}

Tabela 9 - Variação carteira sobrevalorizada

Esta carteira teve um movimento mais semelhante ao do IBOVESPA, coincidindo os anos de alta e de baixa, mas com um rendimento acumulado um pouco melhor que do IBOV, totalizando $234,46 \%$ frente aos $208,45 \%$ do índice. O movimento desta carteira não coincidiu com os resultados esperados, pois era para ela ter um movimento abaixo do IBOV visto que seus papéis eram considerados além do preço justo frente aos dividendos que se estavam pagando, todavia, ainda assim podemos fazer algumas considerações. Esta carteira sobrecomprada também foi montada com ações que pagam dividendos constantemente, ou seja, pelo menos nos 5 anos anteriores à análise e montagem da carteira, ou seja, é um carteira de empresas sólidas, listadas na bolsa e que sempre geram lucro, ou seja, isso por si só já pode ser considerada uma pré seleção de ações com bons fundamentos, já que o único critério para participar do IBOV é ter muita liquidez, podemos ver nele ações de empresas que dão prejuízo mas tem muita movimentação em bolsa, e talvez isso seja um fator que puxa o IBOV para baixo frente à estas ações com maior regularidade de dividendos. Vemos também que o IBOV é preferido em anos de recuperação frente à esta carteira de dividendos, ratificando o mesmo visto na carteira subavaliada e que também a carteira sobrecomprada caiu menos no ano de crise, com uma queda $14,56 \%$ frente à queda de $41,22 \%$ do IBOV. 
Vemos abaixo o gráfico comparativo das 3 carteiras.

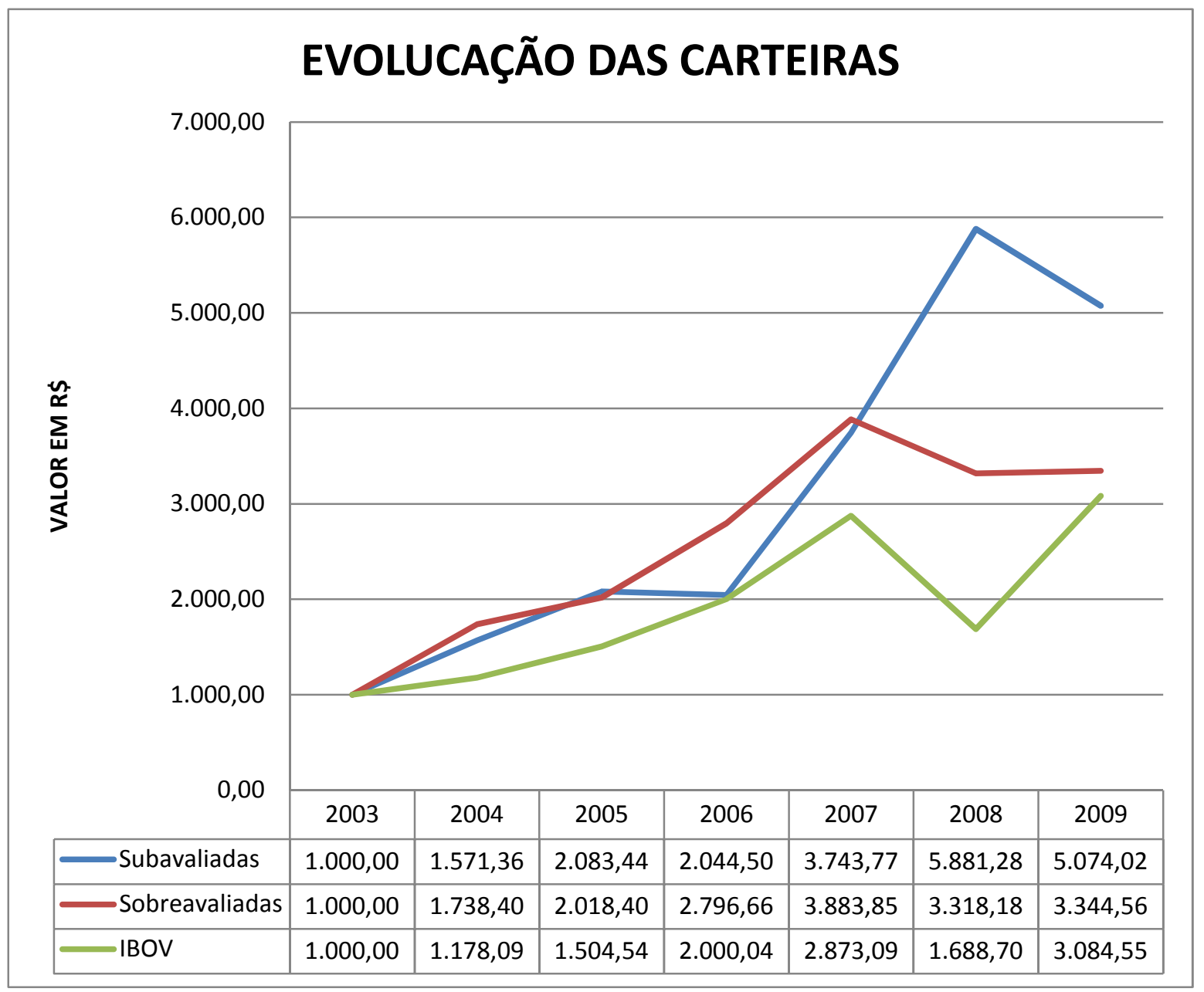

Figura 1 - Comparativo carteiras x IBOV 


\section{CONCLUSÕES E RECOMENDAÇÕES}

Vemos que o método de avaliação de ativos baseado em dividendos pode ser uma alternativa interessante na seleção e avaliação de carteiras e gestão de portfólio. Além de diversificar o risco com um grupo de ações ainda tem-se uma garantia de que estaria investindo em papéis que possuem uma regularidade no pagamento de dividendos aos seus acionistas, o que pode ser uma importante fonte de renda para o investidor comum.

O modelo de fluxo de dividendos descontados também demonstrou-se uma ferramenta mais simples de ser utilizada para o investidor pessoa física, sendo necessário apenas saber os dividendos pagos e taxa de retorno requerida. Pudemos verificar isto neste trabalho onde conseguimos calcular o preço justo para todas as ações que pagam dividendos regularmente em bolsa e fomos selecionando as ações que melhor se encaixavam em nossos critérios, um trabalho que se tornaria impossível de ser feito com o modelo de fluxo de caixa.

O sistema demonstrou-se bastante eficiente na sugestão de carteiras subavaliadas que tiveram uma performance bem acima do índice de referência, sobretudo em momentos de crises. Todavia o sistema não é adequado para sugestão de carteiras sobrecompradas, sendo necessário para este caso algum outro método que não o de dividendos, ou alguma alterações nos parâmetros de cálculos e seleções de papéis propostos.

O Brasil possui algumas peculiaridades que dificultaram a aplicação do modelo, como por exemplo a elevada taxa de juros, que inibe o investimento em bolsa, favorecendo deixar o dinheiro em títulos do tesouro. Segundo WESTSON(2000) “... os níveis de taxa de juros afetam o preço dos ativos". Essas altas taxas aumentam e muito o custo do capital que é formado pela SELIC + Prêmio sobre investimento em ações o que termina diminuindo o preço justo sobre o papel. Além disso, o risco Brasil também coloca um prêmio para investimentos em bolsa sobre os outros países o que também influencia no preço do papel negociado em bolsa. Podemos mencionar ainda, a alta oscilação do índice Bovespa que dificulta o cálculo do prêmio de ações sobre títulos sem risco, talvez por ser um mercado ainda em desenvolvimento. 
Como sugestões para trabalhos futuros, pode-se tentar utilizar o modelo de Gordon com alguma outra fórmula de cálculo de custo de capital e CAPM, conforme sugerido por Assaf Neto:

O Capital Asset Pricing Model (CAPM) oferece conceitos válidos e aceitos na definição do risco dos ativos. Essa metodologia de cálculo é medida pela relação entre a covariância dos retornos de mercado e da companhia e a variância dos retornos da empresa. Por tratar-se de uma metodologia aplicada em mercados estáveis, as conclusões favoráveis ao CAPM precisam, no entanto, ser reavaliadas em mercados emergentes, como o brasileiro [...] (Assaf Neto e outros, 2008, p.1)

O sistema ainda pode ser melhorado e testado talvez excluindo o cálculo do beta das ações ou utilizando algum outro redutor ou ainda executando o cálculo do modelo de Gordon em duas ou três etapas, com uma fase de crescimento acelerado e outra de crescimento menor ou constante. Podemos ainda tentar utilizar uma tabela de prêmio de risco Brasil para utilizar um valor de prêmio sobre ações não constante ano a ano para refletir os períodos de crise. 


\section{REFERÊNCIAS}

BUFFET, M.; CLARK, DAVID. Warren Buffet e a análise de Balanços. Rio de Janeiro: Sextante, 2010.

DAMODARAN, A. Avaliação de investimentos. Ferrametnas e Técnicas para a Determinação do Valor de Qualquer Ativo Rio de Janeiro: QualityMark, 1997.

Bazin, Décio. Faça Fortuna com ações, antes que seja tarde. São Paulo: CLA, 1992.

ROSS, S.; WESTERFIELD, R.; JAFFE, J. Administração Financeira. São Paulo: Atlas, 1995.

WESTSON, J.FRED; BRIGHAM; EUGENE F. Fundamentos de Administração Financeira. São Paulo: Makron Books, 2000.

GITMAN L. JEFFREY Princípios de Administração Financeira. São Paulo: Addison Wesley, 2004.

BM\&FBOVESPA. Empresas/Proventos em dinheiro Disponível em: $<$ http://www.bmfbovespa.com.br/cias-listadas/consultas/proventos-emdinheiro.aspx?Idioma=pt-br >. Acesso em: 24 jun. 2010.

GRAFBOLSA Cotações e gráficos Disponível em: < HTTP://www.grafbolsa.com > Acesso em: 24 jun. 2010.

FUNDAMENTUS Dados de empresas Disponível em: < HTTP://www.fundamentus.com.br >. Acesso em: 21 mai. 2010.

SANTOS, ELIEBER MATEUS; PAMPLONA E. O. Teoria das Opções Reais: Uma atraente opção no processo de análise de investimentos. São Paulo: R.ADM, 2005

NUNES M.I. Análise Fundamentalista Com Foco Para a Recomendação de Compra/Venda de Ações. Up-to-Date no 87, 2004.

NETO, ALEXANDRE ASSAF; LIMA; ARAUJO. Uma proposta metodológica para o cálculo do custo de capital no Brasil. São Paulo, artigo, 2008.

SOLOMON, EZRA; PRINGLE, JOHN. Introdução à Administração Financeira. São Paulo, Atlas, 1981.

MARTELANC, ROY; PASIN, RODRIGO; CAVALCANTE, FRANCISCO. Avaliação de Empresas, um guia para fusões \& aquisições e gestão de valor. São Paulo, Pearson, 2005. 
COPELAND, TOM; KOLLER, TIM; MURRIN, JACK. Avaliação de Empresas "valuation", Calculando e gerenciando o valor das empresas. São Paulo, Makron, 2000.

FERNÁNDEZ, PABLO; Valuations Methods and Shareholder Value Creation. USA, Elsevier, 2002. 